\title{
IMPROVING CHILDREN'S AND THEIR VISITORS' HAND HYGIENE COMPLIANCE
}

\begin{tabular}{|r|l|}
\hline Journal: & Journal of Infection Prevention \\
\hline Manuscript ID & Draft \\
\hline Kanuscript Type: & Original Article \\
\hline Keyword: & $\begin{array}{l}\text { Hand hygiene compliance, children, visitors, educational intervention, } \\
\text { behavioural change, Infection Prevention }\end{array}$ \\
\hline Abstract: & $\begin{array}{l}\text { Background: Numerous interventions have tried to improve healthcare } \\
\text { workers' hand hygiene compliance, however little attention has been paid } \\
\text { to children's and their visitors' compliance. } \\
\text { Aim: To increase children's and visitors' compliance using interactive } \\
\text { educational interventions. } \\
\text { Methods: This was an observational study of hand hygiene compliance } \\
\text { before and after the introduction of educational interventions. Qualitative } \\
\text { data in the form of Questionnaires and interviews was obtained. } \\
\text { Findings: Hand hygiene compliance increased by 21.4\% (P <0.001) } \\
\text { following the educational interventions, with children's compliance reaching } \\
\text { 40.8\% and visitors' being 50.8\%. Compliance varied depending on which } \\
\text { of the five moments of hygiene was observed (P<0.001), with the highest } \\
\text { compliance was 'after body fluid exposure' (96\%). Responses } \\
\text { from questionnaires showed educational interventions raised awareness of } \\
\text { the importance of hand hygiene }(69 \%, 57 \%) \text { compared to those who } \\
\text { hadn't experienced the educational intervention (50\%). } \\
\text { Conclusion: Educational interventions may result in a significant increase in } \\
\text { children's and visitors' hand hygiene (P <0.001). }\end{array}$ \\
\hline
\end{tabular}

\section{SCHOLARONE"}

Manuscripts 
3 Abstract

4 Background: Numerous interventions have tried to improve healthcare workers' hand 5 hygiene compliance, however little attention has been paid to children's and their visitors' 6 compliance.

7 Aim: To increase children's and visitors' compliance using interactive educational 8 interventions.

9 Methods: This was an observational study of hand hygiene compliance before and after 10 the introduction of educational interventions. Qualitative data in the form of Questionnaires 11 and interviews was obtained.

12 Findings: Hand hygiene compliance increased by $21.4 \%(P<0.001)$ following the 13 educational interventions, with children's compliance reaching $40.8 \%$ and visitors' being $1450.8 \%$. Compliance varied depending on which of the five moments of hygiene was 15 observed $(\mathrm{P}<0.001)$, with the highest compliance was 'after body fluid exposure' $(96 \%)$. 16 Responses from questionnaires showed educational interventions raised awareness of 17 the importance of hand hygiene $(69 \%, 57 \%)$ compared to those who hadn't experienced 18 the educational intervention (50\%).

19 Conclusion: Educational interventions may result in a significant increase in children's 20 and visitors' hand hygiene $(\mathrm{P}<0.001)$.

Keywords: Hand hygiene compliance, children, visitors, educational intervention, 24 behavioural change, infection prevention 


\section{Background}

33 Children are vulnerable to infectious diseases (Willmott et al., 2016) and NICE 2017 34 (NICE, 2017) calls for education providers and parents to do more to promote good hand 35 hygiene practices. This is especially relevant when considering children's vulnerability in 36 healthcare settings where not only are children treated by a plethora of healthcare workers 37 who travel in and out of different clinical settings, but they are typically surrounded by ill 38 people. Consequently the healthcare environment has been emphasised as a potential 39 source of harm for patients in the last few years and the reduction of healthcare associated 40 infections (HCAI) is now part of the everyday delivery of healthcare treatment.

41 To prevent and reduce HCAI transmission, it is important to determine if the main routes of 42 exposure to infection are direct, indirect, or due to repeated person-to-person contact. In 43 children, the transmission of infections is likely to correlate with their natural behaviour 44 (e.g. regular exploration of their mouths). The resultant spread of respiratory secretions 45 coupled with an immature immune system combine to increase children's risk of infections 46 (Snow et al., 2008) and they are especially at high risk of respiratory infections and 47 gastrointestinal diseases (Stein et al., 2007).

Hand hygiene $(\mathrm{HH})$ is the single most important measure for reducing $\mathrm{HCAl}$, and interventions can improve compliance (Randle et al., 2010) with the most effective being multimodal.(Naikoba and Hayward, 2001; Gould et al., 2017)

Unsurprisingly studies have focused on Healthcare workers' compliance and patients' and visitors' has been overlooked, even though their Hand Hygiene Compliance (HHC) is 3 important, especially if they augment the care provided by the HCWs as a parent would. 4 Patients and visitors pose a high risk because of their potential to (i) transmit virulent 5 pathogens from the community to the healthcare setting and/or (ii) transfer pathogens 
56 within clinical areas to the patient (directly or indirectly).(Gould et al., 2017; Randle et al.,

57 2010; Munoz-Price et al., 2012)

58 This study monitored children's and their visitors HHC before and after the introduction of

59 an educational intervention (Supplementary Figure A) The educational intervention was

60 either the Glo-yo (Supplementary Figure B, Supplementary Figure C, Supplementary

61 Table A) ; or a video.

62

63 
64

65

66

67

68

69

70

71

72

73

74

75

76

77

78

79

80

81

82

83

84 themes.

\section{Methods}

Ethical and Regulatory Aspects

The Research Ethical Committee (REC) Committee East Midlands Research NHS and the Research \& Innovation department, NHS, approved this study.

\section{Study design}

This observational study was conducted on six paediatric wards in a teaching Hospital in the East Midlands. Random sampling (slips of paper in a hat) allocated two paediatric wards for each educational intervention (the Glo-yo or the video) and the control group which received no intervention (see Supplementary Table A). The baseline phase included HHC rates using the WHO 5 moments of hand hygiene (2009). The intervention phase included Hand $\mathrm{HHC}$ rates and the educational interventions. After the interventions, a qualitative questionnaire was given to the parents/carers of the children (3-15 years) or children ( $\geq 16)$. Questions asked about $\mathrm{HH}$ behaviours, beliefs and attitudes about infection, hygiene and cleanliness that may influence or prevent effective $\mathrm{HH}$, and views about different $\mathrm{HH}$ approaches, including the use of the Glo-yo or Video.

\section{Statistical analysis}

0 The data were analysed using SPSS statistic software (IBM SPSS statistic v. 21) and

1 GraphPad Prism6. HHC rates composed of simple frequency counts and Chi-square 2 tests. The questionnaire responses were collated in categories inherent in the 83 questions themselves, compared using simple frequency counts and grouped into 85 
86

87

\section{Results}

\section{Baseline}

A total of $525 \mathrm{HH}$ opportunities of patients and visitors were monitored, and the overall compliance rate was $157 / 525$ (30\%, Table IA: proportion complied). HHC was low, particularly for children (10\%). This rate was significantly different from that of their visitors (26\%: $\mathrm{P}<0.05)$. There was also a significant difference in $\mathrm{HHC}$ dependent on the moment of $\mathrm{HH}$, irrespective of whether they were children or visitors $(P<0.001)$. The lowest level of compliance was observed after contact with patient surroundings (13\%), and the highest was after exposure to body fluid (100\%). Similarly, HHC of patients and visitors depended on the ward that they were on $(P=0.31)$ and were significantly different dependent on the time of day $(P<0.001)$.

\section{Post intervention phase}

$1437 \mathrm{HH}$ opportunities were observed. $\mathrm{HHC}$ increased by $24.4 \%$ compared to the baseline phase, and was significantly different between (i) children and visitors $(P<0.01)$, (ii) the moments of contact providing the opportunity, (iii) the type of paediatric ward observed, and (iv) the intervention used $(P<0.001)$ (Table IB). The higher $\mathrm{HHC}$ in the afternoon shift was not significantly different from the morning shift $(P=0.29)$. HHC of patients and visitors in both intervention groups (but not the control group) was significantly different to the baseline phase $\mathrm{HHC}(P<0.001)$. The control group had similar $\mathrm{HHC}$ during the intervention phase $(30.1 \%)$ compared to the baseline $(32.3 \%)$. Interestingly $\mathrm{HHC}$ improvement was greatest after the intervention session using the Glo-yo, and this was a statistically significantly difference $(P<0.001)$. 
109

110

111 Of the 62 children and visitors approached, 31 agreed to participate in the educational

112 intervention. The Glo-yo group included 16/31 (51.6\%) of the participants (9/16 were

113 patients). The Video group included $7 / 31$ (22.5\%) of the participants (5/7 patients). The

114 control group included 8/31 (25.8\%) of the participants (1/8 patients) (who only had access

115 to $\mathrm{HHC}$ leaflets). All children were given a questionnaire to complete to determine their 116 perception of the intervention session.

117 Children reported that the educational interventions raised their awareness of hand 118 hygiene, with the Glo-yo intervention prompting a higher proportion of the participants to 119 indicate that they strongly agreed with this (Figure 1).

120

121 The intervention session helped increase children's knowledge and understanding of germs and handwashing

123 The questionnaire sought participant feedback on; A. why we wash our hands, B. germs

124 and bacteria, C. when to wash hands, and D. parts of hands that are difficult to wash. The 125 answers varied between intervention and subcategory of question. The Glo-yo intervention 126 group agreed strongly with respect to all question subcategories (Figure 2).

127 Almost two thirds of participants in the Glo-yo and MLT intervention groups strongly 128 agreed that the session and both training aids focused on why we wash our hands $(62.5 \%$ 129 and $71.4 \%$ ), but $100 \%$ of the control group merely agreed with this (Figure $2 a$ ). When 130 asked about whether the intervention increased knowledge about bacteria and germs, $13133.3 \%$ of the participants in the Glo-yo group highly agreed and $100 \%$ of the Video group 132 agreed, which contrasted with the control group, who were $100 \%$ neutral on this point 133 (Figure 2a). When the participants considered whether the intervention sessions dealt 
134 with when to wash hands, $88 \%$ of the Glo-yo group strongly agreed, whereas $71 \%$ of the

135 Video group and $88 \%$ of control group were neutral (Figure 2c). Finally, when asked

136 whether the intervention session increased the knowledge and understanding of the parts

137 of hands that are difficult to wash, $69 \%$ of the Glo-yo group, $43 \%$ of the Video group and

138 only $13 \%$ of the control group strongly agreed. Indeed, a small proportion of the

139 participants of the Video and controls disagreed with this (Figure 2d).

140

141 The session improved children's handwashing even for one day

142 Due to the limited time that patients spend in hospital, and because the session was only

143 performed once with each participant, the final part of the questionnaire aimed to

144 determine whether a single intervention session could improve handwashing even for one

145 day. More than half of the Glo-yo group strongly agreed $56 \%$ whilst the participants of the

146 other intervention groups were less convinced (Figure 3).

148 Discussion

149 Studies focusing on the HHC of patients and visitors in healthcare settings are limited

150 (Buet et al., 2013). However, previous studies have reported an increase in HHC after

151 educational intervention (McGuckin et al., 1999; Chen and Chiang, 2007; Fishbein et al.,

152 2011).

153 Children and visitors had the highest level of compliance 'after exposure to their own body

154 fluids which has previously been identified (Randle et al., 2010) This may be as a result of 155 self-protection, or due to emotional sensations including feelings of unpleasantness, 156 discomfort and/or disgust (Whitby et al., 2007). The lowest compliance was found for the 157 moment 'after contact with patient surroundings'. This increased after intervention by $45 \%$ 
158 to reach $58.3 \%$. Although this is considered a low compliance rate, it is significantly higher

159 than recent data (Randle et al., 2013), and it is important as near touch sites pose the

160 highest risk to patients, especially those in close and direct contact with patients (Dancer,

161 2009). Another high increase in HHC was observed 'after contact with patients'. This was 162 mainly observed in visitors, increasing from $23.7 \%$ to $70.8 \%$, to reach a level $>20 \%$ higher

163 than previous observational studies (Randle et al., 2010). No study was found that looked

164 at $\mathrm{HHC}$ of patients before a meal, in this study it was observed that compliance at this 165 opportunity at the intervention phase was as high as $65 \%$.

166 This study indicates that $\mathrm{HHC}$ is better than previously reported, and provides evidence of 167 a significant increase in $\mathrm{HHC}$ during intervention $(\mathrm{P}<0.001)$. The Glo-yo session proved

168 the most successful intervention and was able to raise awareness of the importance of

$169 \mathrm{HH}$, with parents strongly agreeing that the Glo-yo session will improve their child's hand 170 washing. This aligns with previous research indicating educational and psychological 171 programmes integrating tangible materials and images of the subject to be learnt can 172 improve motivation and learning with the added benefit of long term behavioural change

173 (Bairaktarova et al., 2011; Worthington et al., 2001; Ho et al., 2009).

174

175 Acknowledgements

176 Thanks to: ward managers and the clinical lead for children's services at Nottingham

177 University Hospitals Trust, healthcare workers, children and their families.

178 The Authors declares that there is no conflict of interest.

179

180

\section{References}


181

182

183

184

185

186

187

188

189

190

191

192

193

194

195

196

197

198

199

200

201

202

203

204

205

206

207

208

209

210

211

212

213

214

215

216

217

218

219

220

221

222

223

224

225

Bairaktarova D, Evangelou D, Bagiati A, et al. (2011) Early Engineering in Young Children's Exploratory Play with Tangible Materials. Children, Youth and Environments 21: 212-235.

Buet A, Cohen B, Marine M, et al. (2013) Hand Hygiene Opportunities in Pediatric Extended Care Facilities. Journal of Pediatric Nursing-Nursing Care of Children \& Families 28: 72-76.

Chen YC and Chiang LC. (2007) Effectiveness of hand-washing teaching programs for families of children in paediatric intensive care units. Journal of Clinical Nursing 16: 1173-1179.

Dancer SJ. (2009) The role of environmental cleaning in the control of hospital-acquired infection. Journal of Hospital Infection 73: 378-385.

Fishbein AB, Tellez I, Lin H, et al. (2011) Glow Gel Hand Washing in the Waiting Room: A Novel Approach to Improving Hand Hygiene Education. Infection Control and Hospital Epidemiology 32: 661-666.

Gould DJ, Moralejo D, Drey N, et al. (2017) Interventions to improve hand hygiene compliance in patient care. Cochrane Database of Systematic Reviews.

Ho JH, Zhou SZ, Wei D, et al. (2009) Investigating the Effects of Educational Game with Wii Remote on Outcomes of Learning. In: Pan Z, Cheok AD, Muller W, et al. (eds) Transactions on Edutainment lii. 240-252.

McGuckin M, Waterman R, Porten L, et al. (1999) Patient education model for increasing handwashing compliance. American Journal of Infection Control 27: 309-314.

Munoz-Price LS, Birnbach DJ, Lubarsky DA, et al. (2012) Decreasing Operating Room Environmental Pathogen Contamination through Improved Cleaning Practice. Infection Control and Hospital Epidemiology 33: 897-904.

Naikoba S and Hayward A. (2001) The effectiveness of interventions aimed at increasing handwashing in healthcare workers - a systematic review. Journal of Hospital Infection 47: 173-180.

NICE. (2017) Healthcare-associated infections: prevention and control in primary and community care. NICE Guidance.

Randle J, Arthur A and Vaughan N. (2010) Twenty-four-hour observational study of hospital hand hygiene compliance. Journal of Hospital Infection 76: 252-255.

Randle J, Firth J and Vaughan N. (2013) An observational study of hand hygiene compliance in paediatric wards. Journal of Clinical Nursing 22: 2586-2592.

Snow M, White GL and Kim HS. (2008) Inexpensive and time-efficient hand hygiene interventions increase elementary school children's hand hygiene rates. Journal of School Health 78: 230-233.

Stein C, Kuchenmuller T, Hendrickx S, et al. (2007) The Global Burden of Disease Assessments WHO Is Responsible? Plos Neglected Tropical Diseases 1.

Whitby M, Pessoa-Silva CL, McLaws M, et al. (2007) Behavioural considerations for hand hygiene practices: the basic building blocks. Journal of Hospital Infection 65: 1-8.

Willmott M, Nicholson A, Busse H, et al. (2016) Effectiveness of hand hygiene interventions in reducing illness absence among children in educational settings: a systematic review and meta-analysis. Archives of Disease in Childhood 101: 42-50.

Worthington HV, Hill KB, Mooney J, et al. (2001) A cluster randomized controlled trial of a dental health education program for 10-year-old children. Journal of Public Health Dentistry 61: 22-27. 
227

228

229

230

231

232

233

234

235

236

237

238

239

240

241

242

243

244

245

246

247

248

249

250

251

252

253

254

Figure 1. Participant feedback indicated that interactive sessions raised their awareness of the importance of Handwashing.

Figure 2. Participants agreed that the intervention sessions helped increase children's knowledge and understanding of germs and handwashing. The responses to the second question on the questionnaire are shown ('The session helped increase your child's knowledge/understanding of the following:').

Figure 3. Participants agreed that the intervention sessions would be effective in improving children's handwashing even for one day, with the strongest positive response being for the Glo-yo intervention.

Table 1. $A x^{2}$-Test of difference in proportions of opportunities adhered to, across levels of variable. Left Column (A) shows the baseline data and right Column (B) the intervention (intermidate phase data)

*significant $) \mathrm{P}<0.05)$

${ }^{* *}$ highly significant $(P<0.001)$

Table 2. Participant comments in response to two of the questions on the questionnaire.

Supplementary Material:

Supplementary Figure A. An outline of the study

Supplementary Figure B. Glo-yo interactive educational toy.

Supplementary Figure C. The leaflet distributed in the control group

Supplementary Table A. Comparative description of the training aids used in the intervention phase of the trial. 


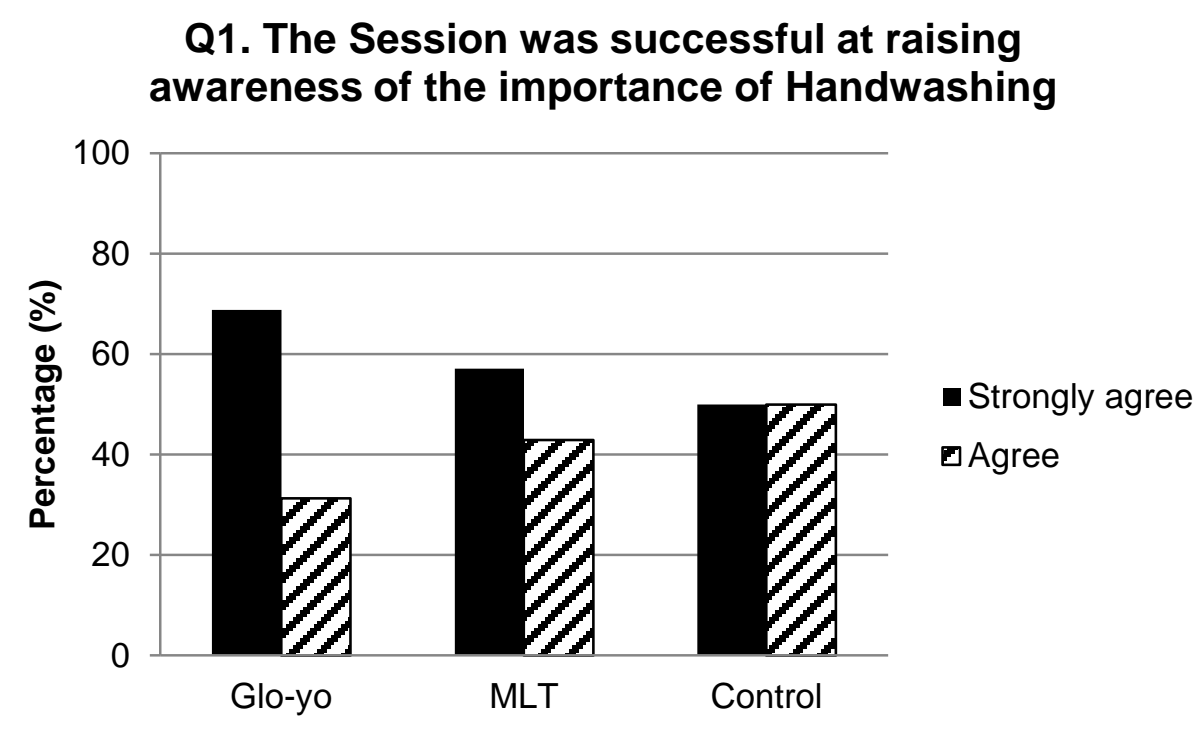

Figure 1 
Q2. The session helped increase your child's knowledge/understanding of the following:

a. Why we wash hands

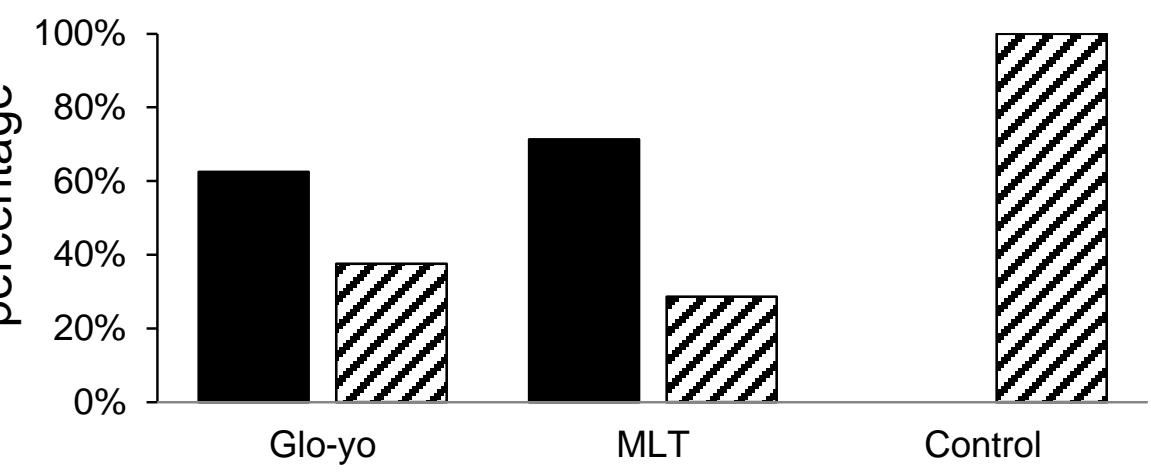

c. When to wash hands

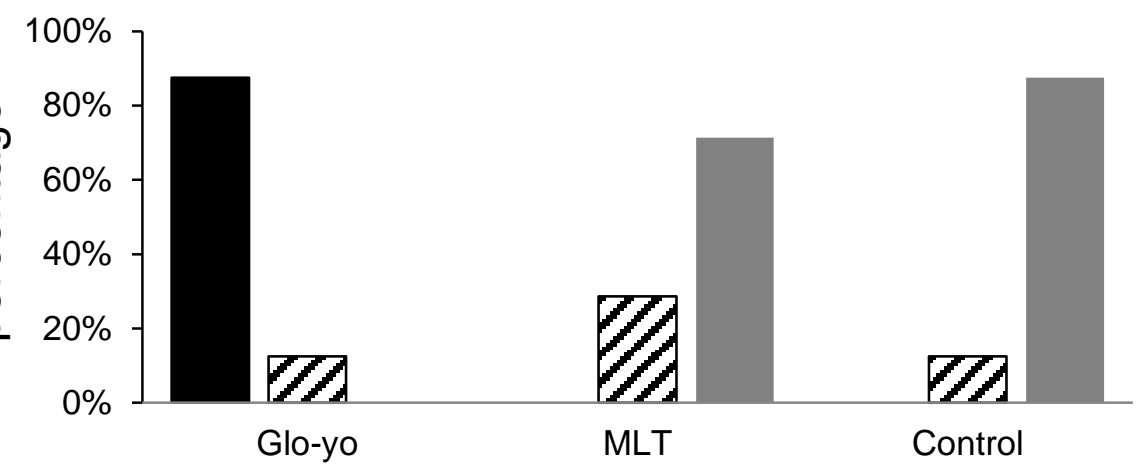

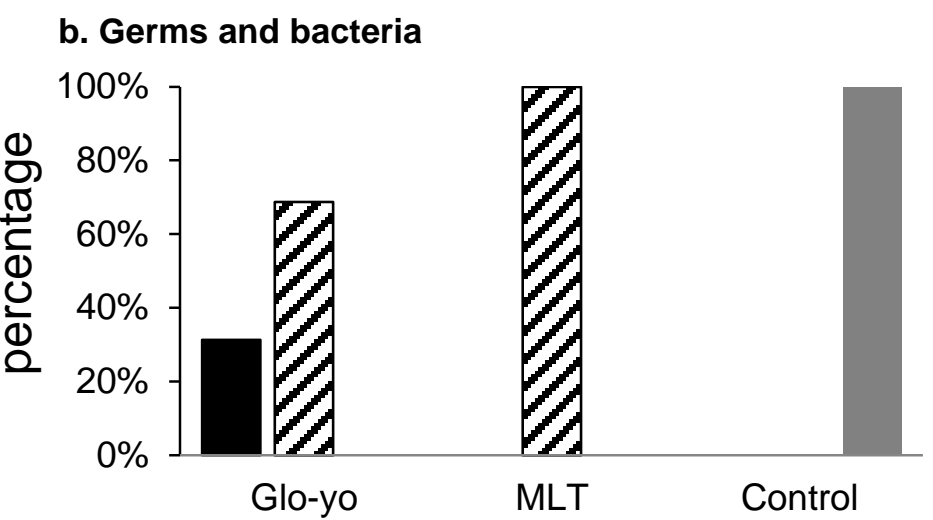

d. Parts of hands that are difficult to wash

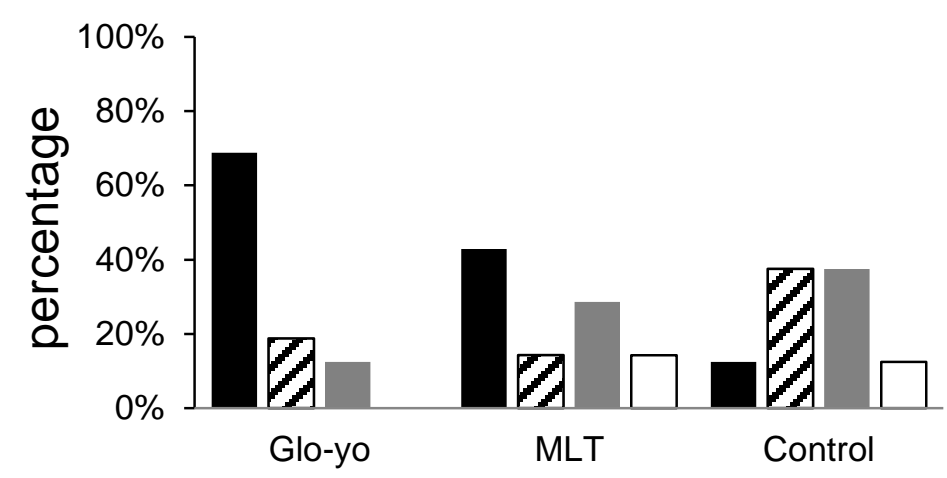




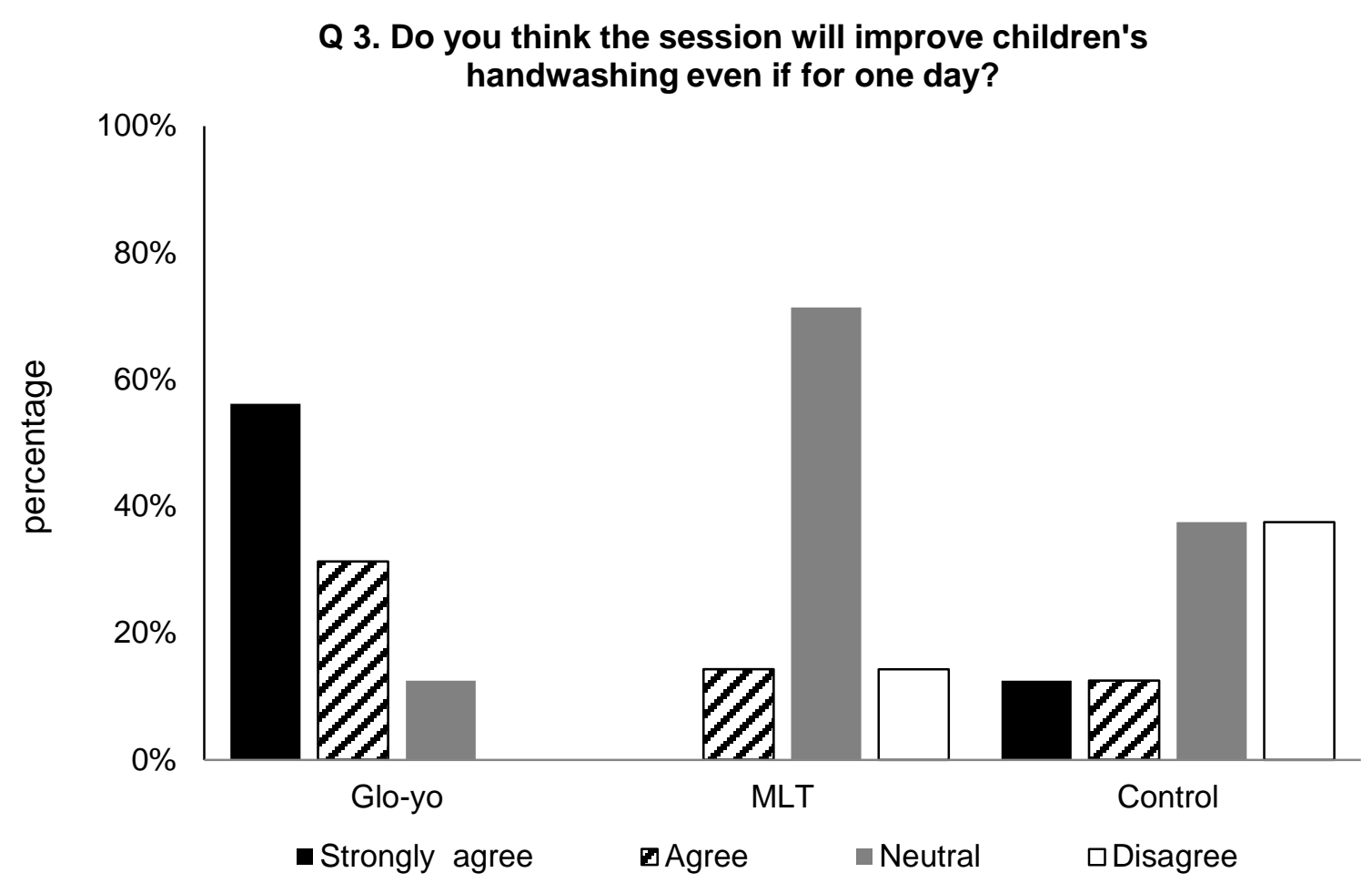

Figure 3 


\section{A. Baseline}

\section{B. Intervention}

\begin{tabular}{|c|c|c|c|c|c|c|c|c|c|c|}
\hline Variable & \multicolumn{2}{|c|}{$\begin{array}{l}\text { No. (\%) } \\
\text { Proportion }\end{array}$} & \multicolumn{2}{|c|}{$\begin{array}{l}\text { Proportion } \\
\text { complied }\end{array}$} & $P=$ value $^{\mathrm{a}}$ & \multicolumn{2}{|c|}{ No. (\%) Proportion } & \multicolumn{2}{|l|}{$\begin{array}{l}\text { Proportion } \\
\text { complied }\end{array}$} & \multirow[t]{2}{*}{$P=$ value $^{\mathrm{a}}$} \\
\hline A. Patients and visitors & 525) & & & & & B. Patie & and Visitc & S $\quad(N=1437$ & & \\
\hline Patients & 33 & (6.3) & $3 / 33$ & (9.9) & $<0.05^{*}$ & 228 & $(15.9)$ & $93 / 228$ & $(40.8)$ & $<0.5$ \\
\hline Visitors & 492 & $(93.7)$ & $127 / 492$ & $(25.8)$ & & 1209 & $(84.1)$ & $614 / 1209$ & $(50.8)$ & \\
\hline \multicolumn{11}{|l|}{ Ward } \\
\hline General paediatric & 129 & $(24.6)$ & $25 / 129$ & (19.3) & 0.31 & 340 & $(24.6)$ & $156 / 340$ & $(45.9)$ & $<0.001^{* *}$ \\
\hline Surgical ward & 96 & (18.3) & $27 / 96$ & $(28.1)$ & & 319 & $(20.6)$ & $190 / 319$ & $(59.6)$ & \\
\hline Dialysis and urology & 33 & (6.3) & $5 / 33$ & (15.1) & & 147 & (11.6) & $85 / 147$ & $(57.8)$ & \\
\hline Oncology & 97 & $(18.5)$ & $29 / 97$ & $(29.8)$ & & 198 & $(13.5)$ & $95 / 198$ & $(48.8)$ & \\
\hline Neuroscience & 119 & $(22.7)$ & $32 / 119$ & $(26.8)$ & & 198 & $(15.1)$ & $96 / 198$ & $(48.5)$ & \\
\hline Children's assessment & 51 & $(9.7)$ & $12 / 51$ & $(23.5)$ & & 231 & $(14.6)$ & $85 / 231$ & $(36.8)$ & \\
\hline Time & & & & 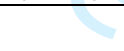 & 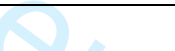 & & & & & \\
\hline Morning & 270 & (51.4) & $49 / 270$ & $(18.1)$ & $<0.001^{* *}$ & 689 & $(47.9)$ & $349 / 689$ & $(60.6)$ & 0.29 \\
\hline Afternoon & 255 & $(48.6)$ & $81 / 255$ & (31.7) & & 748 & $(52.1)$ & $358 / 748$ & $(62.3)$ & \\
\hline \multicolumn{11}{|l|}{ Type of opportunity } \\
\hline Before patient contact & 188 & (35.8) & $76 / 188$ & (40.4) & $<0.001^{* *}$ & 1943 & $(47.5)$ & $1136 / 1943$ & $(58.4)$ & $<0.001^{* *}$ \\
\hline $\begin{array}{l}\text { After body fluid } \\
\text { exposure risk }\end{array}$ & 31 & (5.9) & $31 / 31$ & (100) & & 25 & $(<1)$ & $24 / 25$ & (96) & \\
\hline After patient contact & 122 & $(23.2)$ & $29 / 122$ & (23.7) & & 1026 & $(25.1)$ & $726 / 1026$ & $(70.8)$ & \\
\hline $\begin{array}{l}\text { After contact with } \\
\text { patient surroundings }\end{array}$ & 184 & $(35)$ & $24 / 184$ & (13) & & 1051 & $(25.7)$ & $696 / 1051$ & $(58.3)$ & \\
\hline Before meal & & & & & & 43 & (1) & $28 / 43$ & $(65.1)$ & \\
\hline \multicolumn{11}{|l|}{ Intervention Group } \\
\hline Control & 170 & $(32.3)$ & $44 / 170$ & $(25.8)$ & $<0.001^{* *}$ & 433 & $(30.1)$ & $181 / 433$ & $(25.6)$ & $<0.001^{* *}$ \\
\hline Glo-yo & 129 & $(24.5)$ & $32 / 129$ & $(24.8)$ & & 466 & $(32.4)$ & $275 / 466$ & (59) & \\
\hline MLT & 226 & (43) & $54 / 226$ & $(23.8)$ & & 538 & $(37.4)$ & $251 / 538$ & $(46.7)$ & \\
\hline
\end{tabular}


Table 1. A $x^{2}$-Test of difference in proportions of opportunities adhered to, across levels of variable. Left Column (A) shows the baseline data and right Column (B) the intervention (intermidate phase data)

*significant $) \mathrm{P}<0.05$ )

**highly significant $(P<0.001)$ 
Table 2. Participant comments in response to two of the questions on the questionnaire.

'The session in general is encouraging for children to learn about hand washing and germs. The use of phone is not easy for my child to understand'

'The video is too long and not easy to

MLT follow by child'

'The video is very complicated for a child to understand. I enjoyed the video as it's easier to follow and understand'.

\section{Comments on the question \\ 'Would you recommend the sessions to children in schools'}

'Strongly agree, schools will be much better to learn about hand washing'

'Very interesting would like to have same session in schools'

'Mum found it interesting and very worthwhile. I would recommend it to school children. My daughter found it interesting'.

I would recommend the hand wash in schools it will encourage children more to wash hands and be germ free than hospital'.

'For my child was too much information, $i$ enjoyed the video as a parent'.

'The session was encouraging!'

'The session with the facilitator was fun hand washing session was useful. However the phone video is not easy for child to understand'.

'Session was helpful in understanding when to wash hands, and how, will recommend for school children'.

\section{Control}

'Helpful session should be more practical to apply in schools'. 


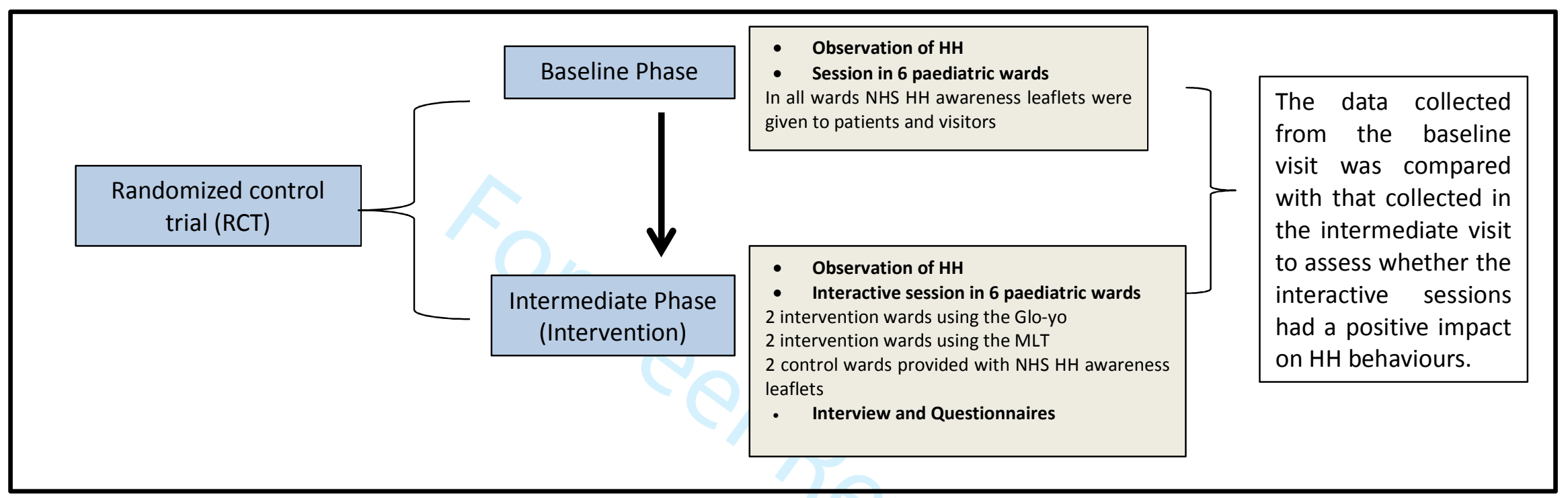

Supplementary Figure A. 
a

b

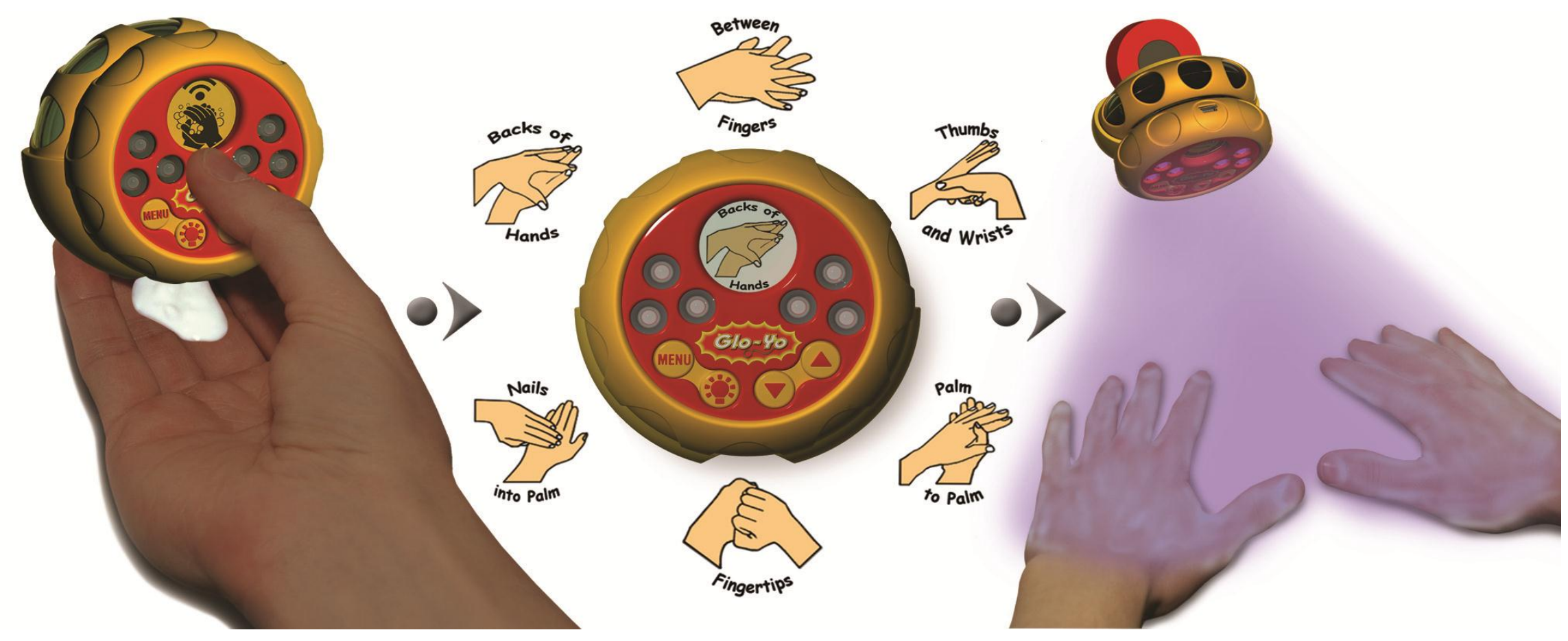

Supplementary Figure B. Glo-yo interactive educational toy. (a) handheld Glo-yo, (b) 6 images of the HHC steps displayed on the screen during 20 seconds, (c) UV lights illuminate the iridescent cream on hands as a way to assess the effectiveness of HHC [14]. 


\section{Hand Hygiene}

"Hand Washing is the single most important method of preventing and controlling infection."

Health Protection Agency

To try to minimise the impact of infections in hospitals it is important that all staff, visitors and patients wash their hands on a regular basis.

Studies have shown that when people wash their hands there are areas that are regularly missed, these are shown by the red areas on the picture opposite. To try to prevent this it is recommended, that, when washing your hands you use the six

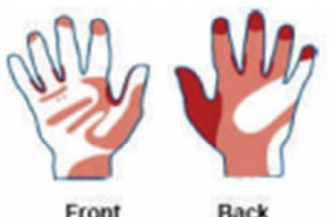
step process outlined below.

Whilst hand washing is the most effective method of preventing hospital acquired infections it is recognised that access to a hand wash basin is not always easy, in these cases use of alcohol hand sanitiser may be used instead. Again it is recommended that the same six step process should be used.

If you see others not washing their hands, politely ask them to do so!
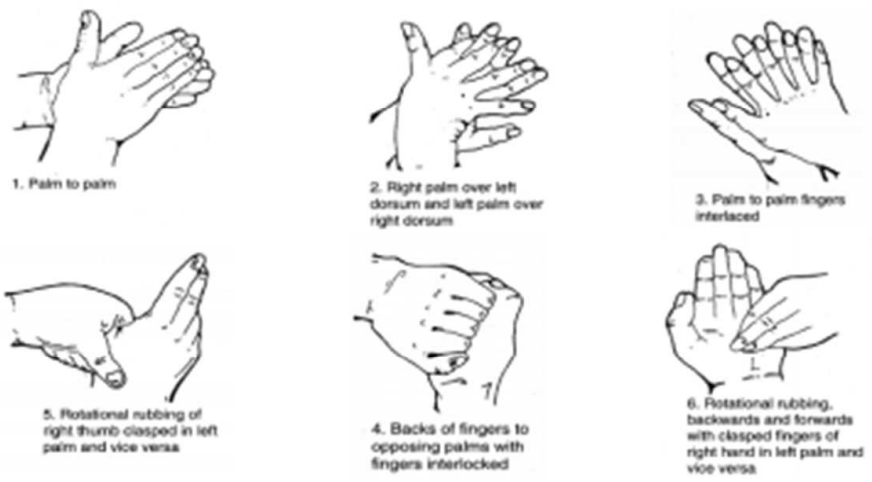


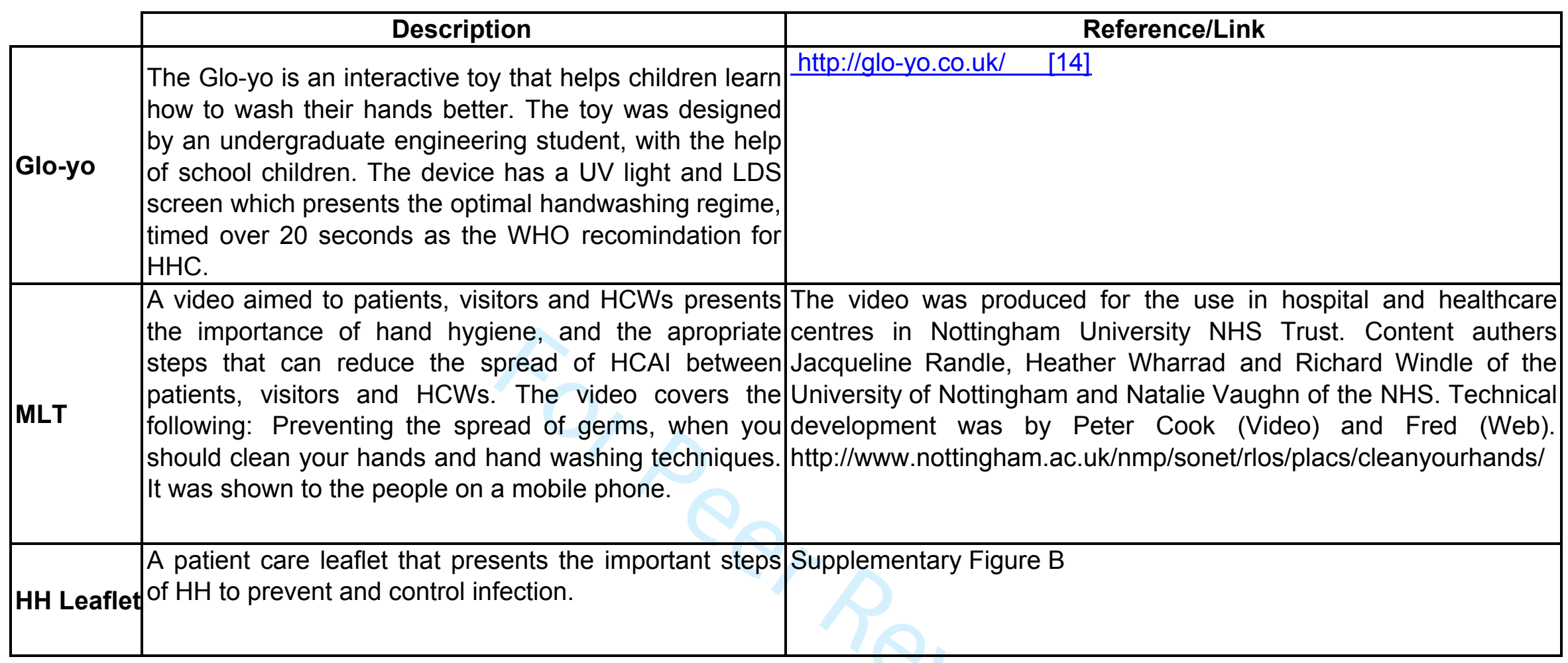

Supplementary Table A. Description of the Glo-yo, MLT and leaflet used for the RCT. 